\title{
Determinants of maternal infection associated with virus infection of the fetus and newborn infant
} William J Britt

\author{
Address: Depts of Pediatrics, Microbiology, and Neurobiology, University of Alabama School of Medicine, Birmingham, AL, USA \\ from Fourth Dominique Dormont International Conference. Host-Pathogen Interactions in Chronic Infections \\ Paris, France. 13-15 December 2007 \\ Published: 9 April 2008 \\ Retrovirology 2008, 5(SuppI I):L3 doi:I0.I I86/I742-4690-5-SI-L3
}

This abstract is available from: http://www.retrovirology.com/content/5/SI/L3

(c) 2008 Britt; licensee BioMed Central Ltd.

Congenital infection with human cytomegalovirus (HCMV) is most common intrauterine acquired virus infection in infants in the developed world. Rates of congenital HCMV infection range from $0.2 \%-2.0 \%$ in live births. Although maternal infection with this virus rarely results in clinical symptoms, approximately $10 \%$ infants born with congenital HCMV infection can exhibit symptomatology ranging from mild hepatitis to severe multiorgan dysfunction, including damaging central nervous system infection. CNS infection can result in microcephaly, retinitis, and abnormalities in psychomotor function. More commonly, infants are clinically asymptomatic at birth but between $10-20 \%$ of infected infants will exhibit long term deficits in neurological function, with hearing loss being the most common long-term sequelae. Congenital HCMV infection is thought to be the most common non-familial cause of hearing loss. Although these characteristics of congenital HCMV infection are well described in studies from the developed world, little is known about the natural history of this infection in resource constrained countries. However, several studies have demonstrated that the incidence of congenital HCMV infection increases with increasing maternal infection. Recent findings from Brazil and India are consistent with these earlier studies and indicate that congenital HCMV infection occurs in 1-2\% of newborn infants in most of the world's populations. Because HCMV infection is universal in these maternal populations and infection is acquired early in life, these findings indicate that maternal immunity to this virus is insufficient to prevent infection and perhaps can only modulate the incidence of disease in infected infants. Studies from the US have demonstrated that infected infants born to women with preconceptional immunity exhibit rates of developmental abnormalities similar to those infants born to women with primary infection acquired during pregnancy. The mechanisms that account for the inability of maternal immunity to prevent infection and limit disease in the developing fetus infected with HCMV have not been defined, but reinfection with new strains of virus has been shown to occur in normal host following community exposure. Reinfection of previously infected hosts with new strains of virus is common in animal models and recent studies in normal women suggest that rates of reinfection could exceed 20\% per annum. HCMV has been shown to encode a number of viral functions that can evade both innate and adaptive immunity in-vitro and in experimental animal models. Although antiviral antibodies have been shown to neutralize virus in-vitro, strain dependent virus neutralizing antibody responses have been well described and appear to arise from minor sequence variation (1-3\%) in some envelop glycoproteins such as $\mathrm{gB}$ whereas significant variations of $>25 \%$ have been reported in other envelop glycoproteins $(\mathrm{gN})$. Whether these differences account for reinfection with different strains of HCMV and contribute to disease in congenitally infected infants remains to be determined. However, the apparent contribution of reinfection to the natural history of this congenital infection in the developing world suggests that HCMV could be a significant cause of morbidity in infants in resource-constrained countries as well as in the developed world. 